\title{
Tangence
}

\section{Les emblèmes de la maladie. Dialogue du corps et de l'âme}

\section{Frédéric Charbonneau}

Numéro 60, mai 1999

L'éloquence du corps sous l'Ancien Régime

URI : https://id.erudit.org/iderudit/008084ar

DOI : https://doi.org/10.7202/008084ar

Aller au sommaire du numéro

Éditeur(s)

Presses de l'Université du Québec

ISSN

0226-9554 (imprimé)

1710-0305 (numérique)

Découvrir la revue

Citer cet article

Charbonneau, F. (1999). Les emblèmes de la maladie. Dialogue du corps et de l'âme. Tangence, (60), 105-118. https://doi.org/10.7202/008084ar d'utilisation que vous pouvez consulter en ligne.

https://apropos.erudit.org/fr/usagers/politique-dutilisation/ 


\section{Les emblèmes de la maladie. Dialogue du corps et de l'âme}

Frédéric Charbonneau, Université McGill

Pour Stephen Dinsmore

Nous voulons proposer ici une méditation sur l'éloquence du corps - non pas celui de l'orateur rompu aux méthodes de l'actio, mais bien du corps en général, considéré lui-même en quelque sorte comme orateur, dicendi peritus, parlant sa langue propre et de son propre chef.

Or, sans doute, qu'il fût profane ou sacré, tribun, magistrat ou prélat, l'homme public trouvait dans les temps de crise un moment favorable à l'exercice de la parole: les philippiques, les prêches s'élevaient au-dessus d'enceintes fiévreuses ou navrées, dans des cités que le malheur frappait, et s'employant, selon les cas, à attiser l'ardeur des peuples ou à les purger de la corruption. De même, il semblerait que certains parlassent davantage et mieux dans l'état critique de la maladie que dans la paix du tempérament. Ainsi, l'on sait avec Aristote que tel déséquilibre des humeurs, que l'excès de mélancolie chaude "est à l'origine des états d'euthymie accompagnés de chants, des accès de folie, et des éruptions d'ulcères et autres maux de cette espèce", mais qu'il dispose également à la faconde :

[B]eaucoup, pour la raison que la chaleur se trouve proche du lieu de la pensée, sont saisis des maladies de la folie ou de l'enthousiasme. Ce qui explique les Sibylles, les Bacis, et tous ceux qui sont inspirés des dieux [...] Et Maracus le Syracusain était encore meilleur poète dans ses accès de folie. ${ }^{1}$

Les manifestations d'enthousiasme offrent à l'examen un corps que le dieu visite, agité par ce qui lui octroie le don de prophétie, la transe étroitement liée à la crainte qu'elle inspire et à la puissance persuasive de l'oracle. La Sibylle abandonne un instant son corps, s'absente, laissant place à Phobus qui en

1 Aristote, L’homme de génie et la mélancolie. Problème XXX, 1, Paris, Rivages, 1988 [Éd. Jackie Pigeaud], 
prend possession comme d'une chaire inoccupée et parle à travers lui. Il ne s'agit donc pas d'une maladie banale, mais d'une extase (ekstasis), d'une sortie de soi qui est la folie sacrée. Les fièvres ordinaires ont-elles ce privilège ${ }^{2}$ ?

Non, sans doute; et pourtant, nous sommes bien d'avis que le corps parle haut pendant la maladie. Sans être en proie à la fureur, sans qu'il soit déserté par l'âme, néanmoins il s'exprime alors seul, ne se contentant pas d'apporter à l'esprit son concours, de lui prêter sa voix et ses gestes; il acquiert une autonomie grâce à laquelle il peut tenir, avec une force particulière, un discours moral sur l'être dont il est le siège. Au reste, depuis l'Antiquité, une discipline aux frontières de la médecine, promise à une longévité exceptionnelle, la physiognomonie - suivant le titre d'un traité du pseudo-Aristote —, avait pris pour objet l'étude des signes physiques qui manifestent les passions, le caractère et les mours $^{3}$. Et bien que, selon cette doctrine, il fût possible en tout temps de lire le corps à livre ouvert, que la maladie n'y fût point d'un apport nécessaire, l'art conjectural qu'elle sous-tendait reçut au début du XvII siècle un double infléchissement qui l'alliait plus étroitement à la science médicale. Dans The Advancement of Learning, Bacon avait écrit en effet qu'il fallait débarrasser la physiognomonie du métissage des pratiques divinatoires; il lui avait reproché en outre d'ignorer les

2 La thèse aristotélicienne sur la prophétie fut d'ailleurs contestée, dès l'Antiquité, en vertu de ce raisonnement, comme le souligne Jackie Pigeaud dans La maladie de l'âme, Paris, Société d'Édition, coll. "Les Belles Lettres", 1981, p. 263. Voir surtout Cicéron, De divinatione, I, XXXVIII, 81, qui voyait dans "la divination le fait en effet d'un esprit intègre, non d'un corps souffrant".

3 Alessandro Fontana, préfaçant Camillo Baldi, La lettre déchiffrée (1622), Paris, Société d'Édition "Les Belles Lettres", coll. "Le corps éloquent", 1993, p. 31 : "Le postulat est celui-là même qui informe toute la médecine antique: la correspondance symétrique et réciproque entre le physique et le moral, entre l'âme et le corps. [...] Pour cette doctrine, le corps se présente comme une sorte de livre où il est toujours possible de lire la nature et les mœurs des individus par le visage, la voix, la forme du nez, les yeux, le front, etc.". Ce postulat est particulièrement net chez les monistes, tels Chrysippe. Voir, par exemple, Jackie Pigeaud, op. cit., p. 368: "Chrysippe a conçu l'âme et le corps comme un recto-verso indissociable, ce qui signifie en gros que lorsque j'ai l'un, j'ai l'autre, lorsque j'ai le jugement[,] j'ai la manifestation physiologique. La titillation, la morsure, le gonflement, l'affaissement, tout cet univers de l'émotivité que Chrysippe a magnifiquement décrit, ne sont pas des conséquences de jugements, mais leur versant physique." 
gestes, les postures et les états momentanés ${ }^{4}$. Par souci de rigueur, par intérêt croissant pour les secrètes altérations de l'être, la vieille discipline hippocratique allait ainsi devenir une méthode moderne de déchiffrement cautionnée par la Faculté, "la mécanique des signes visibles pren[ant] appui sur une dynamique des mouvements cachés " 5 , un art diagnostique par lequel on remonte du symptôme jusqu'au mal.

Or, si l'on admet que le corps peut dire les passions alors même que le courtisan ou l'"homme du monde", en pleine possession de ses moyens, s'efforce à les dissimuler ainsi que le prescrivent la prudence et l'urbanité, combien plus les doit-il révéler lorsque, malade, ce même corps échappe à son emprise. D'autant que passions et maladies ont en commun le péril qu'elles présentent à l'exercice de la raison et au contrôle de soi - leur racine à toutes deux, pathos, dénotant comme chacun sait la "passivité" propre à celui qu'elles secouent. Cette affinité essentielle justifiait le traitement réservé aux passions par les stö̈ciens qui, les définissant comme des "folies brèves", insanice breves, semblaient en renvoyer l'étude à la nosologie ${ }^{6}$. De même,

4 "[Physiognomy and exposition of natural dreams] have of later time been used to be coupled with superstitions and fantastical arts, yet being purged and restored to their true state, they have both of them a solid ground in nature [...]. In the former of these I note a deficience. For Aristotle hath very ingeniously and diligently handled the factures of the body, but not the gestures of the body, which are no less comprehensible by art, and of greater use and advantage. For the lineaments of the body do disclose the disposition and inclination of the mind in general; but the motions of the countenance and parts do not only so, but do further disclose the present humour and state of the mind and will" (Francis Bacon, The Advancement of Learning (1623), London, Dent, 1962 (1915), p. 107 [éd. G. W. Kitchin]).

5 A. Fontana, loc. cit., p. 37.

6 Voir, entre autres, la célèbre ouverture du De ira de Sénèque: "Ut autem scias non esse sanos quos ira possedit, ipsum illorum habitum intuere [...]: flagrant et micant oculi, multus ore toto rubor exæstuante ab imis præcordiis sanguine, labia quatiuntur, dentes comprimuntur, horrent ac surriguntur capilli, spiritus coactus ac stridens, articulorum se ipsos torquentium sonus, gemitus mugitusque et parum explanatis vocibus sermo præruptus et complosæ sæpius manus et pulsata humus pedibus et totum concitum corpus magnasque minas agens, foeda visu et horrenda facies depravantium se atque intumescentium". "Veux-tu te rendre compte que celui-là en effet a perdu la raison, qui est possédé par la colère? Considère son aspect extérieur. [...] l'œil s'allume et brille, tout le visage se colore sous l'afflux du sang venu du coeur, les lèvres claquent, les dents se serrent, les cheveux se hérissent, la respiration est pénible et sifflante, les articulations se tordent et 
l'impératif de retenue qui fait le courtisan — et plus généralement l'homme civilisé ${ }^{7}$ - lui impose face aux passions, aux souffrances morales, à l'excessive sensibilité, une méfiance qui s'exprime, dans telle maxime de Gracián, par des métaphores de blessures et de chairs douloureuses:

Ne point montrer le doigt malade. Car chacun y viendra frapper. Garde-toi aussi de t'en plaindre, d'autant que la malice attaque toujours par l'endroit le plus faible; le ressentiment ne sert qu'à l'exciter. Elle ne cherche qu'à jeter hors de ses gonds ( $V a$ buscando la mala intención el achaque de hazer saltar), elle coule des mots piquants, \& met tout en œuvre, jusqu'à ce qu'elle ait trouvé le vif. L'homme adroit ne doit donc jamais découvrir son mal, soit personnel, soit héréditaire, attendu que la Fortune même se plaît quelquefois à blesser à l'endroit, où elle sait que la douleur sera plus aiguë. Elle mortifie toujours au vif, \& par conséquent il ne faut laisser connaître ni ce qui mortifie, ni ce qui vivifie; pour faire finir l'un, \& faire durer l'autre (Gracián, L’homme de cour, frag. 145). ${ }^{8}$

Cette équation, qui décèle quelque terrible sympathie, le lexique vient encore la souligner, certains des mots par lesquels on énonce la maladie annonçant, dirait-on, l'état moral par équivoque: la langueur, par exemple, est définie par Furetière comme une "Diminution des forces, de santé, causée par une maladie lente, \& souvent mortelle", mais "Se dit aussi en Morale des tristesses, des afflictions, ou des passions violentes qui nous privent de joye, ou de santé". Observons dans ce dernier trait le lien de causalité unissant le moral au physique, étiologie à propos de

craquent, des gémissements, des mugissements se font entendre, la phrase est coupée de mots indistinctement prononcés, les mains se heurtent, les pieds battent le sol, tout le corps est agité et prend une allure menaçante; le visage, laid et repoussant, se déforme et se gonfle" (Traités philosophiques, Paris, Garnier, s.d., t. 1 [éd. F. et P. Richard]).

7 Nous renvoyons à l'ouvrage désormais classique de Norbert Elias, Über den Prozess der Zivilisation, trad. fr. en 2 tomes, "La civilisation des mœurs" et "La dynamique de l'Occident", Paris, Calmann-Lévy, 1973-1975 (1939).

8 Balthasar Gracián, Oráculo manual y arte de prudencia (1647). Nous citons dans la traduction d'Amelot de la Houssaie (1684). Cf. ibid., frag. 98, 126, 173,287 , passim. Notons que le terme "achaque", qui au figuré signifie "prétexte", a également le sens de "maladie" qu'ont retenu certains traducteurs; ainsi en est-il de The Art of Worldly Wisdom, dans la traduction de Joseph Jacobs (1892), New York, Frederick Ungar, 1967: "Ill-will searches for wounds to irritate". On est tenté de dire qu'illustrant son propos, Gracián a caché la "maladie" sous le "prétexte". 
laquelle La Rochefoucauld naguère s'était livré à un exercice de virtuosité :

L'ambition a produit les fièvres aiguës et frénétiques; l'envie a produit la jaunisse et l'insomnie; c'est de la paresse que viennent les léthargies, les paralysies et les langueurs; la colère a fait les étouffements, les ébullitions de sang, et les inflammations de poitrine; la peur a fait les battements de coeur et les syncopes; la vanité a fait les folies; l'avarice, la teigne et la gale; la tristesse a fait le scorbut; la cruauté, la pierre; la calomnie et les faux rapports ont répandu la rougeole, la petite vérole, et le pourpre, et on doit à la jalousie la gangrène, la peste, et la rage. ${ }^{9}$

Du point de vue de l'observateur, le corollaire de ce qui précède est que le récit de la maladie, que le portrait du corps souffrant implique un jugement sur les mœurs du propriétaire; non seulement, en effet, ce corps malade les trahit par une affinité foncière que la culture de l'honnête homme ne masque plus; mais davantage encore, la langue même par laquelle on dépeint la maladie paraît à double entente. L'alliance de l'éthique et du pathologique serait donc radicale, ce qui ne veut pas dire simple. Si éloquent qu'il soit, le discours du corps moribond attend son interprétation et fait entrevoir l'âme, speculum in cenigmate.

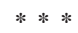

Soit le récit de la mort de Descartes par Adrien Baillet: après avoir marqué les symptômes de la maladie qui devait l'emporter, "quelques frissons... suivis d'une fièvre continue avec une inflammation de poumon" ${ }^{10}$, le biographe consacre plusieurs pages à

9 La Rochefoucauld, "Réflexions diverses", XII, dans Euvres complètes, Paris, Gallimard, coll. "Bibliothèque de la Pléiade", 1950, p. 519 ]éd. crit. L. MartinChauffier].

10 Adrien Baillet, "La mort de Monsieur Descartes" (1691), dans Euvres et lettres de Descartes, Paris, Gallimard, coll. "Bibliothèque de la Pléiade", 1953, p. 1405 et sqq. [éd. crit. A. Bridoux]. On sait que la médecine classique distinguait les fièvres selon l'humeur d'où elles provenaient, selon le temps qu'elles duraient et selon la périodicité de leurs accès. La fièvre continue comptait elle-même quatre espèces: la synoque simple (sanguine), la quotidienne continue (pituiteuse), la tierce continue (bilieuse, dont les accès viennent un jour sur deux) et la quarte continue (mélancolique, dont les accès viennent deux fois en quatre jours). 
110

l'examen de ses causes réelles et supposées. Nous retenons les plus significatives pour notre propos:

L'origine de sa maladie, tout évidente qu'elle ait été à ceux qui vivaient avec lui, n'a pas laissé d'être enveloppée de nuages et d'obscurités par divers esprits brouillons [...]. Les [uns] ont publié que sa maladie lui était venue d'une prise excessive de vin d'Espagne qui lui avança ses jours, et qui rendit sa fin semblable à celle de Démocrite, auquel il n'avait pu souffrir qu'on le comparât durant sa vie. Selon la fiction de quelques autres, M. Descartes voulant traiter sa goutte (qui est un mal chimérique dont il n'eut jamais la moindre atteinte) suivant ses principes, et s'imaginant qu'elle ne venait que faute du mouvement de la matière sublime (ces Messieurs ont voulu dire subtile sans doute), il s'échauffa le sang, qu'il tâchait de rendre plus fluide en se gorgeant d'eau-de-vie. D'autres ont inventé que la Reine Christine, n'ayant pu entrer dans ses pensées, ni goûter ses nouvelles opinions, ne l'avait pas beaucoup considéré, et que le chagrin qu'il en avait conçu lui avait tellement serré le coeur qu'il en contracta le mal auquel il fallut succomber. ${ }^{11}$

L'intérêt de ces trois lectures est dans la pluralité de jugements qu'elles manifestent: la malveillance interprète diversement les signes à la faveur des souplesses du discours médical et, passant de la fièvre aux chaleurs de l'alcool ou de l'irritation, voit dans la maladie du philosophe le discours involontaire de l'intempérance, de la présomption ou de l'aigreur, voire de la débauche - une cause possible de la goutte. Le corps est ambigu; loin d'imposer un sens, il en suggère plusieurs et parfois d'opposés, donnant naissance à des thèses qu'ensuite on défendra, engendrant en quelque manière une éloquence seconde. Cette ambiguité, et l'insistance avec laquelle on s'efforce de la fixer, nous arrêterons quelques moments, afin de préciser ce qui permet ou embarrasse l'interprétation du discours moral que paraissent tenir les corps malades.

Prenons en guise d'exemple ce passage des Confessions où Rousseau répond à celui qui dans Le sentiment des citoyens l'accuse "d'être usé de débauche, pourri de vérole, et d'autres gentillesses semblables" en tirant argument d'une malformation: au pamphlétaire qui lui fait un crime de maux physiques et moraux également honteux, l'ermite rétorque en faisant flèche de son infirmité,

11 Adrien Baillet, "La vie de M. Des-Cartes ", extrait reproduit dans ibid., p. $1403-1414$. 
soutenant que non seulement il n'a eu de sa vie "la moindre atteinte d'aucun mal de cette espéce, mais que des gens de l'art [l']ont même cru conformé de maniére à n'en pouvoir contracter" ${ }^{12}$. La maladie ne décèle donc pas forcément les passions ou les mauvaises mours, et l'on n'était pas sitôt condamné qu'atteint; car dans l'esprit du malade, il pouvait arriver que le vice moral fût passé tout entier dans le vice physique: le mal s'épuisant dans le corps, alambic distillant son venin, il laisse l'âme de Rousseau immaculée.

Il est même des cas où la maladie est signe d'élection, à l'instar des fléaux de Job ou de Tobie. Ainsi Agrippa d'Aubigné, persuadé d'avoir été choisi par Dieu pour mener la sainte Réforme, prouvait le caractère sacré de sa mission par des prophéties, par des visions que garantissaient les fièvres; liée aux épreuves physiques et morales par la disgrâce politique qu'elle entraîne, la maladie l'incite à se comparer au "bouc du désert", victime expiatoire de l'Ancien Testament ${ }^{13}$. Cependant, quoique

12 Rousseau, Confessions, XII (1770), dans Euvres complètes, Paris, Gallimard, coll. Bibliothèque de la Pléiade", t. 1, 1959, p. 632 [éd. crit. B. Gagnebin et M. Raymond]. "Le défaut congénital qui le singularise et le condamne aux longues souffrances, l'aide à repousser les accusations infamantes. Contre ceux qui le déclarent "pourri de vérole", Rousseau se fait un allié de sa maladie" (Jean Starobinski, "La maladie de Rousseau", Yale French Studies, $\mathrm{n}^{\mathrm{O}} 28$ (1962), repris dans Jean-Jacques Rousseau. La transparence et l'obstacle, suivi de Sept essais sur Rousseau, Paris, Gallimard, coll. "Bibliothèque des Idées ", 1971, p. 441). Dans le même ordre d'idées, on trouve chez Restif de la Bretonne, rousseauiste à ses heures, cette curieuse notation qui attribue à la débauche le pouvoir de rendre le corps moins vulnérable: "[Babet] était une Fille absolument perdue, qui s'était livrée par pur libertinage à un Hommemarié, qu'elle avait trompé de tout son pouvoir. [...] Ce fut elle qui acheva de corrompre Pauline. Elles accouchèrent dans la même quinzaine, se portèrent beaucoup mieux que des Femmes-honnêtes, \& à-peine rétablies, songèrent à se livrer à tous leurs goûts" (Les contemporaines (1780-1792), $1^{\text {re }}$ Suite, vol. III, nouvelle XIX, "La fille séduite", Leipzig (Paris), Büschel, 1781, p. 226).

13 Aubigné, Sa vie à ses enfans (1630), Paris, Société des Textes Français Modernes, 1986, p. 51 [éd. crit. G. Schrenck]: "En cest aage [six ans] Aubigné veillant dedans son lict pour attendre son precepteur, ouit entrer dans la chambre, et puis en la ruelle de son lict, quelque personne de quy les vestements frottoyent contre les rideaux, lesquels il veit tirer aussi tost, et une femme fort blanche, qui luy ayant donné un baiser froit comme glace se disparut. Morel arrivé le trouva ayant perdu la parole: et ce qui fit depuis croire le rapport de telle vision fut une fiebvre continue qui luy dura quatorze jours". Sur le "bouc du désert" (Lévitique 16, 10.20-22), voir p. 160161. Cf. Isaïe, 53, 4: "Or, ce sont nos maladies qu'il [le Serviteur de Yahvé] portait, nos douleurs dont il prenait la charge, et nous, nous l'estimions frappé, atteint par Dieu et humilié". 
le statut de la maladie comme épreuve de la foi soit attestée depuis les temps vétéro-testamentaires, en règle générale, le point de vue de l'orthodoxie chrétienne est plus prudent: effet de l'intervention divine, la maladie tient lieu à la fois d'expiation et d'avertissement. Dans la mesure où elle châtie, elle atteste la présence du mal; et, par le moyen de la crainte, elle met le pécheur sur la voie de la contrition ${ }^{14}$. C'est l'optique adoptée par Huet dans ses Mémoires:

Pour moi, bien qu'un si grand exemple [celui de saint Augustin] m'excitât depuis longtemps à nettoyer les souillures de ma vie passée, une cause plus grave encore m’y détermina tout à coup. Ce fut une maladie aiguë et presque mortelle qui m'éprouva cruellement durant six mois entiers, et dont je ne suis pas tout à fait remis, encore qu'elle ait cessé depuis quelques années. L'avertissement était dur et sévère mais les effets en furent excellents. Je sentis que Dieu m'appelait à faire mon examen de conscience et à lui offrir mes fautes avec tous les sentiments de la plus profonde humilité. ${ }^{15}$

14 Cf. par exemple Boèce, pour qui l'homme absolument saint serait invulnérable à la maladie parce qu'inaccessible au mal moral: "Est alius cunctis virtutibus absolutus sanctusque ac deo proximus; hunc contingi quibuslibet adversis nefas providentia iudicat adeo ut ne corporeis quidem

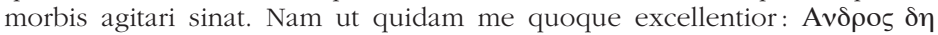
$\iota \varepsilon \rho о v \delta \varepsilon \mu \alpha \varsigma \alpha \iota \theta \varepsilon \rho \varepsilon \varsigma \omega \kappa о \delta о \mu \eta \sigma \alpha v " . ~ " S u p p o s o n s$ l'existence d'un autre homme, en tout point vertueux, saint et très proche de Dieu : l'idée qu'un tel homme puisse être atteint par n'importe quel malheur, apparaît si sacrilège à la Providence qu'elle ne permet même pas à la moindre maladie du corps de venir le tourmenter. Comme le dit en effet quelqu'un d'encore plus éminent que moi: "Les cieux ont bâti la charpente du saint" (IV, 140-145). De Philosophice consolatione, Paris, Rivages, 1989 [trad. C. Lazam]. Le vers grec cité par la Philosophie est incertain et d'origine obscure; la tradition l'attribue à un Père de l'Église. Sur la position du clergé romain, voir le Dictionnaire de spiritualité ascétique et mystique, t. 10, Paris, Beauchesne, 1980, col. 138-139, s.v. "maladie". Notons à cet égard que les évangiles marquent un infléchissement doctrinal, le Christ "se refusant à expliquer l'infirmité ou la maladie par le péché personnel (Jean, 9, 2-3)", col. 140; il n'en demeure pas moins que l'Église, dans sa Glose ordinaire, distinguait cinq effets spirituels de la maladie: "elle augmente le mérite, comme pour Job; elle maintient dans l'humilité, comme [pour] saint Paul; elle vise à la correction de nos péchés, comme pour le paralytique [Matthieu, 9, 2]; elle sert à la manifestation de la gloire de Dieu, par exemple dans le cas de l'aveugle-né; enfin, comme pour Hérode, elle est le début du châtiment mérité ", col. 143.

15 Pierre-Daniel Huet, Mémoires (Commentarius de rebus ad eum pertinentibus, 1718), Toulouse, Société de littératures classiques, 1993, p. 3 [éd. P. J. Salazar]. Voir aussi VI, p. 153: "Le huitième jour, j'étais condamné et abandonné des 
Par la formulation qu'il en donne, l'évêque d'Avranches marque une équivalence entre l'ordure dont il doit déterger sa vie morale (abstergendas pristince vitce sordes) et le "virus" dont une sudation "salutaire" le purge; la brûlure des membres successive à cette opération préfigurant les souffrances par lesquelles l'âme se purifie - et se sauve. Appel à l'auscultation de soi, la maladie est aux yeux du croyant un signe de Dieu, un outil de sa Providence, presque une incarnation de son jugement. Or, vox populi, vox Dei: il arrive que le public se fasse l'organe d'une telle sentence. Remarquable à cet égard est la fin des Liaisons dangereuses, qui, jetant aux orties toute précaution herméneutique, interprète la maladie de $\mathrm{M}^{\text {me }}$ de Merteuil comme la traduction directe et littérale de ses mours.

J'avais bien raison de dire que ce serait peut-être un bonheur pour elle de mourir de sa petite vérole. Elle en est revenue, il est vrai, mais affreusement défigurée; et elle y a particulièrement perdu un œil. Vous jugez bien que je ne l'ai pas revue: mais on m'a dit qu'elle était vraiment hideuse.

Le Marquis de ****, qui ne perd pas l'occasion de dire une méchanceté, disait hier, en parlant d'elle, que la maladie l'avait retournée, et qu'à présent son âme était sur sa figure. Malheureusement tout le monde trouva que l'expression était juste. ${ }^{16}$

La vérole fleurit de pustules le visage de la libertine, montrant par transparence l'infamie, laissant sur son passage des stigmates qui valent une condamnation publique ${ }^{17}$. L'œil surtout paraît

médecins. Ma raison s'en était allée, je n'avais plus sentiment de rien. [...] lorsque, par la grâce de ce Dieu et par un suprême effort de la nature, une sueur salutaire sortit en abondance de toutes les parties de mon corps, entraînant avec soi tout le virus de la maladie, et me sauva de la mort. Je n'étais pourtant pas encore tout à fait guéri; je souffris longtemps de si vives douleurs qu'il me semblait que mes membres brûlaient."

16 Laclos, Les liaisons dangereuses, Lettre CLXXV).

17 Par contraste avec le caractère essentiel de la maladie que suppose le texte de Laclos, citons encore cette page d'un philosophe, lui-même déformé par la vérole, où elle est présentée comme un masque accidentel de la nature, tout comme les attitudes apprises en sont les masques intentionnels : "La physionomie est l'expression du caractère et celle du tempérament. Une sotte physionomie est celle qui n'exprime que la complexion, comme un tempérament robuste, etc.; mais il ne faut jamais juger sur la physionomie: car il y a tant de traits mâles sur le visage et dans le maintien des hommes, que cela peut souvent confondre; sans parler des accidents qui défigurent les traits naturels, et qui empêchent que l'âme ne s'y manifeste, comme la petite vérole, la maigreur, etc.". Vauvenargues, Introduction à la connais- 
114

entériner par son éclipse l'atroce épigramme rapportée par $\mathrm{M}^{\text {me }}$ de Volanges: chez celle dont l'âme fait éruption, point d'usage du "miroir". Habile machiniste, le romancier fait concourir le procès perdu, les intrigues éventées et la maladie à la ruine de la marquise, assurant ainsi l'éradication juridique autant que politique et physique de celle qui enrobait la suppuration morale des charmes d'une sexualité déceptive et maîtrisée.

Des passions dont le corps malade se fait le délateur - et parfois l'avocat - l'amour est celle qui sévit le plus dans la littérature de l'âge classique; elle suffirait seule à prouver l'ambivalence éthique de la maladie, condamnant ou absolvant selon les cas. En effet, suivant la formule de La Rochefoucauld, l'amour est cause des plus grands maux comme des plus grands biens ${ }^{18}$; attaqué, défendu tour à tour, les débats dont il est l'objet engagent le statut des passions en général. Quand il entraîne la maladie et affecte le corps, celui-ci tire de la peinture des souffrances morales un immense pouvoir de persuasion. Exemple parmi d'autres, dans l'Éléonor d'Yvrée de Catherine Bernard, Mathilde, qui pourtant n'est pas aimée, convainc Éléonor de renoncer à son amant le duc de Misnie par l'éloquence d'une fièvre mortelle ${ }^{19}$. Personnage racinien, ravagé, Mathilde illustre l'injustice de la passion par un chantage qui la rendrait tout à fait odieuse s'il n'avait la maladie pour instrument: pitoyable, elle est à demi excusée. Ce caractère tragique, ni entièrement bon ni entièrement mauvais, dominé par l'absolu d'un amour qui lui fait jalousement préférer le malheur de tous au bonheur d'autrui, ne trouve son accomplissement dans l'égarement du corps que grâce à l'ambiguïté foncière de la maladie: susceptible de jugements contraires, elle en permet l'orageuse cohabitation, la netteté de l'ethos cédant en dernière analyse le pas à l'efficacité du pathos. Faute d'être résolu, le problème moral de la maladie est donc suspendu par l'appréciation pragmatique de son éloquence. Ultimement, c'est la rhétorique elle-même qui est mise en cause, toujours soupçonnée de verser dans la sophistique, d'employer à ses fins des matériaux impurs.

sance de l'esprit bumain (1746), XXXVII, Paris, Société littéraire de France, 1920, p. 116.

18 La Rochefoucauld, "De l'origine des maladies", dans Euvres complètes, op. cit., p. 376.

19 Catherine Bernard, Éléonor d'Yvrée (1687), Euvres, t. 1 "Romans et nouvelles", Fasano-Paris, Schena-Nizet, 1993, p. 212 et sqq. [éd. F. Piva]. 
Complémentaire et symétrique de l'âme, le corps malade parle un langage à la fois équivoque et clair. Les dissensions concernant la mort de Descartes ou l'infirmité de Rousseau n'ont d'égale que l'évidence dont se réclame chaque lecture singulière. Les termes du problème sont donc constants: on s'accorde pour voir dans la maladie un signe des mours, mais un signe qui demande, pour être correctement entendu, certaine familiarité, certain savoir que tous n'ont pas, une connaissance de l'intérieur posée comme préalable à l'exégèse. C'est que le corps et l'âme forment ensemble un symbole, au sens de l'étymologie grecque (sumbolon): "un objet coupé en deux, dont deux hôtes conservaient chacun une moitié " 20 . L'autorité de l'interprète dépend ainsi de son caractère d'initié; l'éloquence du corps est un ésotérisme.

Intüs, et in cute ${ }^{21}$ inscrit Rousseau en tête du manuscrit des Confessions; il réfute d'ailleurs Perse en le citant, puisque le satirique prétendait connaître son vis-à-vis "à fond et dans la peau", quand le mémorialiste pense que "[p]ersonne au monde ne [le] connoit que [lui] seul" 22 . Cette thèse extrême, ce quasi solipsisme, qui compare la connaissance morale aux perceptions proprioceptives, ramène la couture de l'âme et du corps sous la surface. La symptomatologie ne saurait dans cette perspective suffire à fonder l'analyse. À l'observation exacte du phénomène, aux savoirs cadres eux-mêmes - théologie spéculative, médecine, physiognomonie -, il faut ajouter quelque chose qui ne relève pas de la science, mais de la proximité. Cette connaissance du secret est une condition de lisibilité du corps malade qui n'est pas énoncée comme telle, mais que l'examen de chaque cas semble impliquer, qu'il soit réel ou fictif.

Il est un rapprochement à faire avec le déchiffrage d'autres

20 Anatole Bailly, Abrégé du dictionnaire grec-français, Paris, Hachette, 1901, S.V.

21 Perse, Satires, III, v. 30: "Ego te intus et in cute novi". Nous citons d'après la traduction d'A. Cartault, Paris, Société d'Édition "Les Belles Lettres", 1929, p. 31 .

22 Rousseau, Lettres à Malesherbes, (4 janvier 1762), dans Euvres complètes, op. cit., t. 1, p. 1133. 
symboles, emblèmes ou devises, qui s'avère éclairant pour notre propos; et c'est avec ce parallèle que nous souhaitons conclure. Par devise, le $\mathrm{XvII}^{\mathrm{e}}$ siècle finissant désignait "une embleme qui consiste en la representation de quelque corps naturel, \& en quelque mot qui l'applique en un sens figuré [...]. Le tableau s'appelle le corps, \& le mot l'ame de la devise" (Furetière) ${ }^{23}$. Ce corps et cette âme symboliques sont l'un par rapport à l'autre dans une relation que règle à l'évidence un modèle humain.

Car l'un \& l'autre devant faire un composé, semblable en quelque façon à celuy que la matiere, \& la forme font ensemble ; il est necessaire qu'il y ait de la proportion entre l'un \& l'autre [...]. Cette proportion demande que le Mot convienne au Corps, dont il est l'Ame; \& qu'il luy convienne de sorte, qu'il ne puisse convenir à une autre Figure, non plus que l'ame de l'homme ne peut convenir au corps du lion. [...] La signification du Corps prise separément est imparfaite; celle des Paroles l'est aussi: mais la signification qui resulte de l'une \& de l'autre, est entiere [...]. ${ }^{24}$

À cette complémentarité de l'âme et du corps, qui fait qu'on ne peut les lire séparément, s'ajoute leur caractère énigmatique, justifié par Bouhours en des termes sociaux, "car la Devise estant un symbole ingenieux, elle ne doit pas estre entenduë du peuple; \& il n'y a que les personnes intelligentes qui en doivent penetrer le secret [...] [Il] faut concevoir en mesme temps je ne sçay quoy de mysterieux, \& de clair; ou plûtost quelque chose qui ne soit ni trop clair, ni trop obscur" ${ }^{25}$. Voilà pourquoi les devises sont habituellement formulées par ellipses et en ces langues étrangères que maîtrisent les honnêtes gens - latin, italien ou espagnol.

Comme celui de la maladie, le langage de la devise est ambigu. Non seulement faut-il que l'interprète soit coutumier des

23 Cette défintion, qui voit dans la devise une variété d'emblème, et que nous avons suivie dans l'énoncé de notre titre, n'aurait pas fait l'unanimité parmi les spécialistes, dont une majorité distinguait fortement les deux espèces de symboles. Voir, entre autres, le Père Pierre Le Moyne, s.j., De l'Art des devises, V, v, Paris, Sébastien Mabre-Cramoisy, 1666, p. 220-223. Au sujet des devises et des emblèmes, on peut consulter les travaux de Mario Praz, dont ses Studies in Seventeenth-Century Imagery, Roma, Edizioni di Storia e Letteratura, "Sussidi Eruditi", 1964.

24 Dominique Bouhours, s.j., Entretiens d'Ariste et d'Eugène, VI "Les Devises", $2^{\mathrm{e}}$ éd., Paris, Sébastien Mabre-Cramoisy, 1671, p. 380, 386.

25 Ibid., p. 417 et 433. 
lois qui président à sa confection, qu'il en traduise et en identifie les signes, mais il lui faut encore éclairer l'Âme par le Corps - et réciproquement - pour parvenir à une signification d'ensemble. Et même cette limitation mutuelle ne suffit pas à supprimer toute ambiguité, quelques devises paraissant osciller entre deux interprétations. Ainsi,

Dom Garcia de Tolede Vice-Roy de Catalogne avoit pour la sienne une Boussole tournée vers l'Etoille polaire,

\section{Nunca otra :}

Il vouloit donner à entendre qu'il ne regardoit en toutes ses actions que la gloire de son Prince; ou plûtost qu'il n'auroit jamais d'inclination que pour une seule personne, qui estoit selon Ruscelli, Victoire Colonne d'Arragon, ou selon d'autres, la Comtesse de Colisan. ${ }^{26}$

Amour ou gloire, la passion qui anime le vice-roi n'est pas fixée - ce qui fait d'ailleurs mentir la figure. Dans cette incertitude des mours, le Corps balance, et avec lui le public, qui ne sait ce qu'il faut entendre par cette éloquente déclaration de fermeté. L'aiguille de la boussole désigne de son doigt pointé l'âme de Don Garcia, comme le mal qui le terrassait, celle de l'évêque d'Avranches, avec clarté et, pourtant, avec opacité.

L'herméneutique dont nous avons tenté le repérage n'est plus guère de saison. Matérialiste, l'Occident moderne a isolé le corps pour mieux l'analyser; et si on l'envisageait autrefois dans son rapport avec l'esprit comme partie d'un symbole, ce lien s'est dégradé en style, jusqu'à devenir dans la maladie la métaphore perverse dont Susan Sontag dénonçait naguère encore les effets ${ }^{27}$. L'ontologie classique nous est devenue aussi étrangère que la physique qui la prolongeait. Or l'attention que l'on accordait à l'éloquence du corps prenait son sens dans la perspective où il y avait véritablement participation morale, interdépendance rigoureuse de l'enveloppe charnelle et de l'esprit qui s'y loge. Voilà bien

26 Ibid., p. 518; cf. p. 516: Marie de Médicis avait pris lors de son veuvage "des Cendres chaudes, ou selon quelques Auteurs, de la Chaux vive d'où il sortoit une grande fumée, à cause des eaux qui tomboient dessus, avec ces Paroles, Ardorem extincta testantur vivere flamma: comme pour dire que ses larmes faisoient paroître l'amour qu'elle conservoit pour son mari : \& pour publier à tout le monde que son cœur estoit toujours ardent, quoy-que le feu qui l'avoit enflamé, fût éteint".

27 Susan Sontag, Illness as Metaphor, New York, Random House, 1979. 
la raison pour laquelle la connaissance du malade est essentielle à la juste interprétation de la maladie ${ }^{28}$ : le corps seul est incomplet, de même que l'âme seule, du moins parmi les hommes - rendus à l'autonomie, ce seraient cadavre et fantôme. Le principe qui permet la lecture du corps est ainsi celui même qui la rend équivoque.

28 "J'aurois bien voulu donner à tous ces Modernes, \& à vous tout le premier, le Prince Antiochus à guérir de sa fiévre quarte. Vous sçavez comme je m’y pris, \& comme je découvris par son poux qui s'émut plus qu'à l'ordinaire en la présence de Stratonice, qu'il estoit amoureux de cette belle Reyne, \& que tout son mal venoit de la violence qu'il se faisoit pour cacher sa passion. Cependant je fis une cure aussi difficile \& aussi considérable que celle-là, sans sçavoir que le sang circulast [...]. Il ne s'agissoit point de nouveaux conduits, ny de nouveaux réservoirs; ce qu'il y avoit de plus important à connoistre dans le Malade, c'estoit le cour". Bernard Le Bovier de Fontenelle, "Dialogues des Morts Anciens avec des Modernes. v. Erasistrate, Hervé", Nouveaux Dialogues des Morts (1683), Paris, Société des Textes Français Modernes, 1971, p. 188 [éd. crit. J. Dagen]. 